\title{
Water structuring and collagen adsorption at hydrophilic and hydrophobic silicon surfaces
}

\author{
Daniel J. Cole, ${ }^{1, *}$ Mike C. Payne, ${ }^{1}$ and Lucio Colombi Ciacchi ${ }^{2,3},+$ \\ ${ }^{1}$ Theory of Condensed Matter Group, Cavendish Laboratory, \\ University of Cambridge, J J Thomson Avenue, Cambridge CB3 0HE, UK \\ ${ }^{2}$ Hybrid Materials Interfaces Group, Faculty of Production Engineering \\ and Bremen Centre for Computational Materials Science, \\ University of Bremen, 28359 Bremen, Germany \\ ${ }^{3}$ Fraunhofer Institut für Fertigungstechnik und Angewandte Materialforschung IFAM, \\ Wiener Str. 12, 28359 Bremen, Germany
}

\begin{abstract}
The adsorption of a collagen fragment on both a hydrophobic, hydrogen-terminated and a hydrophilic, natively oxidised Si surface is investigated using all-atom molecular dynamics. While favourable direct protein-surface interactions via localised contact points characterise adhesion to the hydrophilic surface, evenly spread surface/molecule contacts and stabilisation of the helical structure occurs upon adsorption on the hydrophobic surface. In the latter case, we find that adhesion is accompanied by a mutual fit between the hydrophilic/hydrophobic pattern within the protein and the layered water structure at the solid/liquid interface, which may provide an additional driving force to the classic hydrophobic effect.
\end{abstract}


The issue of protein adsorption onto solid surfaces in a wet environment is of great importance for the design and functionalisation of materials in the contexts of biomedical implants, biosensors, antifouling surfaces, pharmaceutical packaging, and many others [1, 2, 3]. One important case is the adsorption of extracellular matrix proteins such as collagen on the surfaces of implanted Si-based microelectromechanical systems [4]. Control over protein adhesion may be achieved by manipulation of surface properties, such as isoelectric point, functional group termination, topography and hydrophobicity [5, 6, 7, 8, 9, 10, 11, 12]. However, the atomistic details of the interactions mediated by these effects are still unclear and are the object of intense experimental and theoretical investigation [13, 14, 15, 16].

In a previous work [17], we have shown that liquid water close to a natively oxidised Si surface presents alternating layers of larger and smaller density with respect to the bulk. These structural features directly influence the strength of mutual adhesion between pairs of Si wafers at room temperature under humid conditions [17]. In a similar way, the adsorption and adhesion of biomolecules to solid surfaces may be significantly determined by the structure of water layers in the surface proximity [18, 19, 20, 21]. In particular, the disruption of the adsorbed water layer by individual amino acids has been shown to have both enthalpic and entropic effects on the free energy of the bound system [22]. In this work we show through all-atom molecular dynamics simulations (MD) [23] that a complex oligopeptide interacts with a Si surface via a mutual fit between its intrinsic hydrophilic/hydrophobic pattern and the structured water layers close to the surface. This may provide a driving force for adsorption at the solid/liquid interface which acts in addition to the classic hydrophobic effect [13, 24].

Specifically, we use MD to investigate the adsorption behaviour of the NC1(84-116) domain of collagen XIV (PDBID: 1b9q), on both a hydrophilic (i.e. natively oxidised [25]) and a hydrophobic (i.e. hydrogen-terminated) $\mathrm{Si}(100)$ surface. The $\alpha$-helical protein conformation used as the starting point for our simulations has been found by circular dichroism measurements to be stabilised by interaction with hydrophobic surfaces [26]. The folded collagen structure presents one predominantly hydrophobic side and a series of hydrophilic basic residues (lysine) clustered on the opposite side. These basic residues form a specific binding site through which collagen XIV interacts with heparin molecules [26]. Here, we consider adsorption of the $\alpha$-helix on solid surfaces with the heparin binding lysine residues pointing towards the solvent, away from the surface. 
In our simulations, water molecules are treated using the TIP3P force field [27] and protein-water and protein-protein interactions are described by the Amber parm99 biomolecular force field [28]. The water solvent and the protein interact with the surface via Coulomb and Lennard-Jones (LJ) interactions. The atomic charges and interatomic interactions for the natively oxidised Si surface are taken from Ref. [17]. The LJ energies $\epsilon_{i}$ and radii $\sigma_{i}$ of the $\mathrm{Si}$ and $\mathrm{O}$ surface atoms are accurately tuned to reproduce the correct heat of immersion of hydrated silica surfaces [17]. In the case of the hydrophobic H-terminated $\mathrm{Si}(100)$ surface, terminal $\mathrm{H}$ atoms are assigned small ESP charges of -0.08 e (as computed by density functional theory), which are compensated by charges of $+0.08 e$ on their neighbouring $\mathrm{Si}$ atoms. For the $\mathrm{H}$ atoms of the hydrophobic surface, we use the same LJ parameters as for hydrophobic $\mathrm{H}$ atoms of hydrocarbon chains. The surface-protein LJ interactions are obtained by combining the surface and biomolecular force field parameters using the standard Lorentz-Berthelot combination rules [29]. We have performed both shorter simulations allowing all atoms of the surfaces to move and longer simulations where the surfaces remain frozen throughout, except for hydroxyl $\mathrm{H}$ atoms on the natively oxidised surface, which are free to rotate as described in Ref. [17]. The water structure at the rigid natively oxidised surface is nearly identical to that obtained for the unconstrained surface, as also reported in [17].

Two simulations are started initially placing the collagen helix a few $\AA$ above two randomly chosen sites of the natively oxidised Si surface [23]. Close contact between the surface and collagen is achieved by performing a 2000 step conjugate gradients minimisation in vacuum. The entire simulation cells are then filled with water molecules and the cell heights are adjusted to obtain the equilibrium TIP3P water density far from the surfaces. The solvated systems are slowly heated to $300 \mathrm{~K}$ applying weak harmonic constraints to the protein and, finally, constant-temperature production MD runs are performed for $24 \mathrm{~ns}$.

The collagen fragment is found to be highly mobile on the hydrophilic surface (Fig. 1 (top)). In the first simulation, the molecular centre of mass initially moves away from the surface, while localised contact (within $3.4 \AA$ of the surface) is maintained via residues 108-112, resulting in an end-on bound conformation. Later a second point of contact via residues $91-93$ is formed. The position of the centre of mass remains stable at approximately $9 \AA$ over the final 10 ns of the simulation, and the root mean square displacement of the backbone atoms of the protein converges to within $1 \AA$. In the second simulation, 
two points of contact are maintained throughout most of the 24 ns simulation, again via the molecular termini (roughly localised in the regions of residues 84-88 and 104-112). Interestingly, residues Leu107 and Arg111 remain in close contact with the surface throughout both simulations via the hydrophobic $\mathrm{CH}_{n}$ part of their side chain groups. The total energy of adsorption averaged over the final $10 \mathrm{~ns}$ of the two simulations is $-22 \pm 7 \mathrm{kcal} / \mathrm{mol}$ with respect to a reference unbound simulation in which collagen is separated from the surface slab by at least $10 \AA$.

A different behaviour is observed in two similar simulations (each lasting $14 \mathrm{~ns}$ ) performed starting with collagen in the same conformation over two different starting positions close to the hydrophobic, H-terminated Si surface. The fragment remains adsorbed to the surface and maintains its helical structure throughout both simulations (Fig. 1 (centre)), again with an average energy of adsorption of $-22 \pm 3 \mathrm{kcal} / \mathrm{mol}$ with respect to a corresponding unbound system. Contrary to the case of the hydrophilic surface, the residues in direct contact with the surface are evenly spread through the central part of the protein (residues 91-108), while the molecular termini (84-90 and 109-116) are less tightly bound.

As visible in the Ramachandran plot computed over the final 10 ns of each simulation (Fig. 2), the backbone dihedral angles of collagen on the hydrophobic surface are tightly clustered around the ideal $\alpha$-helix values $\left(-65^{\circ},-35^{\circ}\right)$. A time-averaged calculation of the $\alpha$-helix content of the protein using the DSSP algorithm [30] reveals that $33 \%$ of the residues have an $\alpha$-helical structure. This is mainly concentrated in the central protein region of residues $93-112$, in qualitative agreement with a structural assignment based on NMR spectroscopy [26]. For comparison, in a separate 14 ns simulation of collagen in bulk water, there is a much wider spread of dihedral angles, with an increase of their occurrence in the $\beta$-sheet region. With respect to the adsorbed case, the average helical content falls to $18 \%$, and the average number of intramolecular hydrogen bonds decreases from 19 to 17 (as computed with a distance cut-off of $3.4 \AA$ and an angular cut-off of $140^{\circ}$ ). On the hydrophilic surface, the degree of unfolding is intermediate between the hydrophobic and unbound structures (Fig. 2) and 20 internal hydrogen bonds are maintained on average.

Although the degree of $\alpha$-helical conformation in bulk water may be over-estimated due to our helical starting structure, the uncoiling observed in the time scale of these simulations appears to be significant. In Ref. [26], it is contended that unfavourable electrostatic interactions between arginyl and lysyl side chains in the region of the heparin binding site 
contribute to helix destabilisation which causes the collagen domain to unfold in water. Indeed, by computing the pairwise electrostatic interactions involving only the primary heparin binding region (residues 97-105), we find that the net electrostatic energy is approximately $30 \mathrm{kcal} / \mathrm{mol}$ less favourable at the hydrophobic surface than in bulk water, where the Coulomb repulsion between charged residues is lowered as a consequence of the helical unfolding.

These results are consistent with the experimental observations that the $\alpha$-helical form of the NC1 collagen XIV fragment adsorbs stably on hydrophobic organic surfaces [26]. Here we observe that collagen remains adsorbed at the hydrophobic Si-H surface for a total of $28 \mathrm{~ns}$ with a favourable net binding energy. On the one hand, displacements of water molecules close to non-polar amino acids following direct interactions with the surface, together with the associated re-arrangement of the solvent hydrogen-bond network, are indeed known to drive adsorption and represent a fundamental part of the so-called hydrophobic attraction [13, 24]. On the other hand, more subtle structural effects arising from the presence of an abrupt solid/liquid interface combined with a favourable arrangement of the adsorbed protein in an ordered hydrophobic/hydrophilic pattern may also contribute to stabilize the adsorption, as we shall see below.

The effects of the molecular water structure on the biomolecular adsorption are studied by analysing the density profiles of water and protein $\mathrm{H}$ atoms along the direction perpendicular to the surface. In the case of water, this is computed by counting the average number of $\mathrm{H}$ atoms present in thin planar slices of width $0.1 \AA$ in separate 1 ns simulations in the absence of collagen. The disruption of the bulk water hydrogen bond network by the two surfaces results in a re-ordering of interfacial water molecules to maximise their interactions with neighbouring molecules and the surface. This is manifested by the structuring of water into layers of higher and lower density with respect to the bulk, as found previously in numerous MD simulations [17, 31, 32],

In the case of the hydrophobic surface (Fig. 3(a), black line), the first peak in the water density is separated from the surface $\mathrm{H}$ atoms (grey lines) by $2.7 \AA$, consistent with weak surface-water interactions of the Lennard-Jones type [33]. Two other maxima are visible at 5.9 and $9.1 \AA$ far from the surface. This surface-induced water layering is preserved in the presence of adsorbed collagen, as evident from the density profile of the 150 water molecules belonging to the first two solvation shells of the protein fragment (dotted line). The first 
three peaks are centred at 2.8, 6.5, and $9.7 \AA$, only slightly shifted from those observed in the absence of the protein. We now compute in the same way the density of $\mathrm{H}$ atoms belonging to collagen, averaged during the MD trajectories described above, discriminating hydrophobic $\mathrm{H}$ atoms (bonded to $\mathrm{C}$ atoms) from hydrophilic $\mathrm{H}$ atoms (bonded to $\mathrm{O}, \mathrm{N}$ or $\mathrm{S}$ atoms). The $\mathrm{H}$ atoms of the protein are clearly structured in alternating regions of higher and lower density, revealing an intrinsic ordering of hydrophobic/hydrophilic residues in the adsorbed helical structure (Fig. 3(a)). Interestingly, the hydrophobic/hydrophilic pattern within the adsorbed protein is commensurate with the water layering oscillations in the surface proximity. Namely, the first water peak is located between the first and second small peaks of the hydrophobic $\mathrm{H}$ atoms, centred at 2.2 and $3.9 \AA$. The latter peak coincides approximately with the first trough in the water density (located at $4.4 \AA$ ). Correspondingly, the large trough in the hydrophobic $\mathrm{H}$ density at $6.2 \AA$ coincides with the second water density peak. Further, the first peak in the hydrophilic $\mathrm{H}$ density is located at $2.8 \AA$, in perfect correspondence with the large first water peak. The broad main peak overlaps with the second water peak and the third peak (corresponding to the cluster of lysine residues) points away from the surface/protein interface into the bulk solution [34].

In the case of the natively oxidised surface, water molecules penetrate into the oxide layer and the main density peak is centred on the surface hydroxyl groups (Fig. 3(b)). In contrast to the clear structuring observed at the hydrophobic surface, both hydrophilic and hydrophobic $\mathrm{H}$ atoms of the protein show relatively spread and less structured density profiles, and the matching between the hydrophilic/hydrophobic patterns in the molecule and the water layer structure is barely visible. Surprisingly, while hydrophilic H atoms of the protein are shielded from the surface by the first high density water peak, the $\mathrm{H}$ atoms of hydrophobic groups are the closest to the surface. This is consistent with our previous finding that predominantly hydrophobic side chain groups remain in close surface contact (see above), bound via van der Waals forces to bridging oxygen atoms of the oxide network, which are hydrophobic compared to the surface silanol groups [17].

Despite the evident ordering of water molecules at the surface/protein interfaces, their mobility within the protein hydration shells remains of the same order of magnitude before and after adsorption. This is shown by the mean residence times of the first shell water molecules surrounding the wet surfaces, the adsorbed proteins and the protein in bulk water, calculated with the method introduced in Ref. [35] (Table 44). As expected, the residence 
times of water molecules in the surface proximity, especially for the hydrophilic case, are higher than the value of 7 ps obtained for bulk water. However, the residence times of water molecules around collagen (80 ps for the dissolved protein, as found for similar systems [35]) are only slightly increased when the protein is adsorbed at the two surfaces.

To summarise, collagen remains bound to the oxidised Si surface via a few terminal residues, the most stable direct interactions with the surface being between hydrophobic side chain groups and hydrophobic surface sites. Individual hydrophilic residues penetrate into the first water layer to form direct hydrogen bonds with the surface for only $20-30 \%$ of the total simulation time. This is consistent with a suggested adsorption model in which proteins weakly bound to hydrophilic surfaces leave the surface-bound hydration layer almost intact and do not affect the surface tension at the solid/liquid interface [24, 36]. In contrast, proteins tend to adsorb with larger contact surface areas to hydrophobic surfaces [5, 11, 12]. In particular, the NC1 domain of collagen XIV has been observed to adsorb stably in an $\alpha$-helical structure on a hydrophobic organic layer [26], as supported by our simulations on the H-terminated Si surface.

We note that an accurate analysis of the individual enthalpy and entropy contributions leading to the observed adsorption behaviour is made difficult by the large oscillation of each separate energy term and the intermittent exchange of counter-ions between surface, protein and bulk water. Extensions of this work will be necessary to calculate the full potential of mean force along possible adsorption/desorption paths at different temperatures [13]. In our analysis so far, we have focused on the structural details governing collagen adsorption on silicon and provided evidence for a strict interplay between the solvent structure at the solid/liquid interface and the intrinsic hydrophobic/hydrophilic pattern within the adsorbate. Whether this effect is specific to systems that are intrinsically chemically commensurate with the water layering, or whether flexible biopolymers may indeed adjust their internal structure to fit the solvent ordering at the solid/liquid interface (in a process reminiscent of the "induced fit" mechanism for supramolecular organisation [37]) is a question that will require further combined theoretical and experimental efforts.

Computer resources were provided by the Cambridge High Performance Computing Service, UK, and by the Center for High Performance Computing (ZIH) at the University of Dresden, Germany. We acknowledge support by the EPSRC, the Deutschen Forschungsgemeinschaft within the Emmy-Noether Programme (CI 144/2-1), and the European Com- 
munity under the HPC-EUROPA project (RII3-CT-2003-506079) and the EU-FP7-NMP Grant 229205 "ADGLASS”.

* Electronic address: djc56@cam.ac.uk

$\dagger$ Electronic address: colombi@hmi.uni-bremen.de

[1] A. C. Richards Grayson et al., Proceedings of the IEEE 92, 6 (2004).

[2] D. Hal Davis, C. S. Giannoulis, R. W. Johnson, and T. A. Desai, Biomaterials 23, 4019 (2002).

[3] R. F. Service, Science 297, 962 (2002).

[4] G. Voskerician et al., Biomaterials 24, 1959 (2003).

[5] L. Bacáková, E. Filová, F. Rypácek, V. Svorcík, and V. Starý, Physiol. Res. 53, S35 (2004).

[6] T. J. Su, J. R. Lu, R. K. Thomas, Z. F. Cui, and J. Penfold, J. Coll. Inter. Sci. 203, 419 (1998).

[7] B. G. Keselowsky, D. M. Collard, and A. J. García, Biomaterials 25, 5947 (2004).

[8] K. Wilson, S. J. Stuart, A. J. García, and R. A. Latour, J. Biomed. Mater. Res. 69A, 686 (2004).

[9] J. H. Kim and S. C. Kim, Biomaterials 23, 2015 (2002).

[10] A. J. García, M. D. Vega, and D. Boettiger, Mol. Biol. Cell 10, 785 (1999).

[11] U. Jönsson, I. Lundström, and I. Rönnberg, J. Coll. Inter. Sci. 117, 127 (1987).

[12] P. B. van Wachem et al., Biomaterials 6, 403 (1985).

[13] D. Horinek et al., Proc. Natl. Acad. Sci. USA 105, 2842 (2008).

[14] G. Raffaini and F. Ganazzoli, Langmuir 20, 3371 (2004).

[15] G. Raffaini and F. Ganazzoli, Macromol. Biosci. 7, 552 (2007).

[16] J. -W Shen, T. Wu, Q. Wang and H. -H Pan, Biomaterials 29, 513 (2008).

[17] D. J. Cole, G. Csányi, M. C. Payne, S. M. Spearing, and L. Colombi Ciacchi, J. Chem. Phys. 127, 204704 (2007).

[18] J. C. Hower, Y. He, M. T. Bernards, and S. Jiang, J. Chem. Phys. 125, 214704 (2006).

[19] A. M. Bujnowski and W. G. Pitt, J. Coll. Inter. Sci. 203, 47 (1998).

[20] A. N. Cormack, R. J. Lewis, and A. H. Goldstein, J. Phys. Chem. B 108, 20408 (2004).

[21] R. Zangi and J. B. F. N. Engberts, J. Am. Chem. Soc. 127, 2272 (2005).

[22] R. A. Latour and L. L. Hench, Biomaterials 23, 4633 (2002). 
[23] Our MD simulations are performed with the AMBER10 [28] package, using an integration time step of 2 fs and the SHAKE algorithm to constrain H-containing bonds. Coulomb interactions are treated using the Particle Mesh Ewald sum, with a real space cut-off of $10 \AA$. The cut-off length for LJ interactions is also set to $10 \AA$. The temperature is controlled by the weakcoupling algorithm with a 2 ps time constant and, during equilibration, the systems are heated at a rate of $50 \mathrm{~K}$ per $100 \mathrm{ps}$. The protein fragments are terminated with $-\mathrm{NH}_{3}^{+}$and ${ }_{-}^{-\mathrm{COO}^{-}}$groups, and seven $\mathrm{Cl}^{-}$ions are added to ensure charge neutrality. $\mathrm{Si}(100)$ surface models of area $95.7 \mathrm{~nm}^{2}$ are obtained by repeating a $(2 \times 2)$ unit cell. The surface slabs are placed in a supercell subject to periodic boundary conditions and separated by a vacuum gap of approximately $60 \AA$. The Si lattice parameter is fixed to the equilibrium value obtained with the Stillinger-Weber potential $(5.44 \AA)$ and the oxide structure is taken from Ref. [25]. Structural and energetic properties are calculated over the final 10 ns in each case. For the non-rigid surface simulations, we use the surface force field described in [17] and the DL_POLY_3 MD package.

[24] E. A. Vogler, Adv. Colloid Interface Sci. 74, 69 (1998).

[25] L. Colombi Ciacchi, D. J. Cole, M. C. Payne, and P. Gumbsch, J. Phys. Chem. C 112, 12077 (2008).

[26] R. Montserret et al., Biochemistry 38, 6479 (1999).

[27] W. L. Jorgensen, J. Chandrasekhar, J. D. Madura, R. W. Impey, and M. L. Klein, J. Chem. Phys. 79, 926 (1983).

[28] D. A. Case et al., Amber10, University of California, San Francisco (2008).

[29] J. S. Rowlinson and F. L. Swinton, Liquids and Liquid Mixtures (Butterworths, London, 1982).

[30] W. Kabsch, C. Sander, Biopolymers 22, 2577 (1983).

[31] C. Y. Lee, J. A. McCammon, P. J. Rossky, J. Chem. Phys. 80, 4448 (1984).

[32] L. Liu, M. Krack, A. Michaelides, J. Am. Chem. Soc. 130, 8572 (2008).

[33] The calculated heat of immersion of the model H-terminated Si surface, $-22 \mathrm{~mJ} / \mathrm{m}^{2}$, is in good agreement with values inferred from contact angle measurements of H-terminated gold surfaces [38].

[34] Notably, the same ordering between the water and collagen structure is observed in shorter simulations $(\sim 2 \mathrm{~ns})$ in which all atoms of the surface are allowed to move.

[35] C. Schröder, T. Rudas, S. Boresch, and O. Steinhauser, J. Chem. Phys. 124, 234907 (2006). 
[36] E. A. Vogler, J. C. Graper, H. W. Sugg, L. M. Lander, and W. J. Brittain, J. Biomed. Mater. Res. 29, 1017 (1995).

[37] M. Lingenfelder, G. Tomba, G. Costantini, L. Colombi Ciacchi, A. De Vita, K. Kern, Angew. Chem. Int. Ed. 46, 4492 (2007).

[38] H. Keller, W. Schrepp, and H. Fuchs, Thin Solid Films 210/211, 799 (1992). 


\section{Figure Captions}

FIG. 1: MD trajectories of collagen on a natively oxidised (top) and a H-terminated (centre) $\mathrm{Si}(100)$ surface and in a separate simulation in bulk water, in the absence of any surface (bottom). Initial (left), intermediate (centre) and final (right) snapshots are pictured. Collagen consists of 34 amino acid residues and its predominantly hydrophobic surface is initially oriented towards the surfaces. Water molecules are omitted for clarity.

FIG. 2: Ramachandran plots showing the distributions of the dihedral angles, $\phi$ and $\psi$ (degrees), for collagen at the hydrophobic surface (left), hydrophilic surface (centre) and in bulk water (right).

FIG. 3: Density profiles of hydrophobic (green) and hydrophilic (blue) H atoms of collagen at (a) a H-terminated and (b) a natively oxidised Si surface. Density profiles of water H atoms (black, solid line) are obtained from separate $1 \mathrm{~ns}$ simulations. Also shown are the $\mathrm{H}$ atoms of water molecules belonging to the first two solvation shells of collagen (black, dotted line) and $\mathrm{H}$ atoms of the surfaces (grey).

FIG. 4: Water mean residence times (ps) about the protein adsorbed at the hydrophobic and hydrophilic surfaces and about the protein in bulk water. Also shown are the residence times of water molecules at the two surfaces in the absence of collagen. The two values quoted at hydrophilic surfaces are for hydroxyl groups and bridging oxygen atoms respectively. Snapshots are analysed every 2 ps in sliding windows of $400 \mathrm{ps}$ in the final $10 \mathrm{~ns}$ of each simulation. The mean residence times are obtained by fitting a stretched exponential to the autocorrelation functions of the boolean variable that indicates whether or not a given water molecule is within a shell of width $3.5 \AA$ around the protein or surface. 
Hydrophilic

$$
0 \mathrm{~ns}
$$

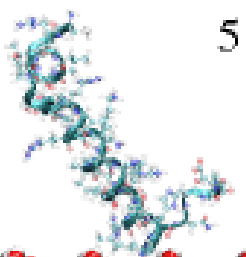

(1)

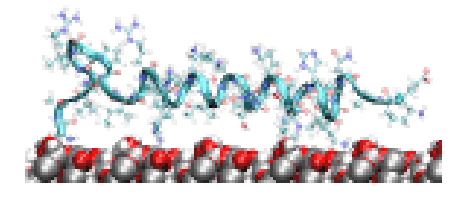

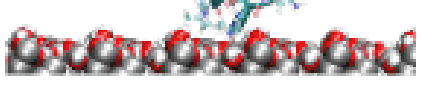

$5 \mathrm{~ns}$

$24 \mathrm{~ns}$

(2)

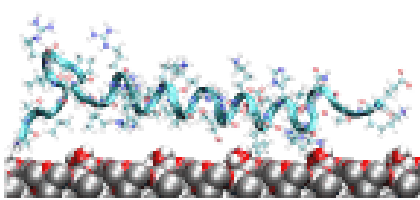
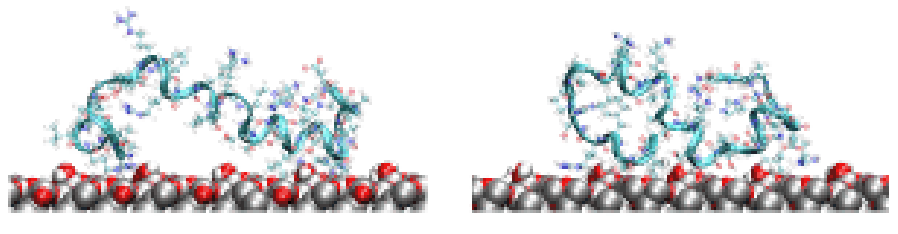

Hydrophobic

$0 \mathrm{~ns}$

$5 \mathrm{~ns}$

$14 \mathrm{~ns}$

(1)

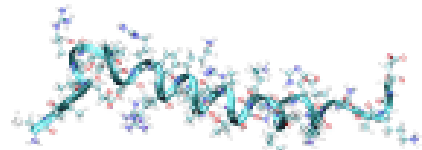

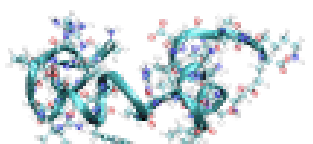

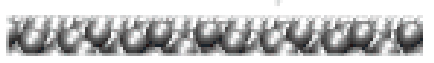

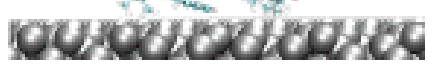

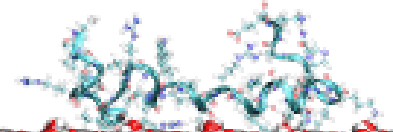

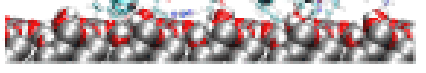

(2)

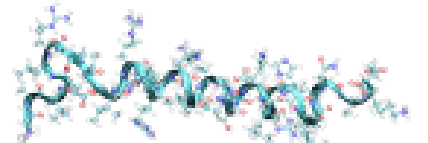

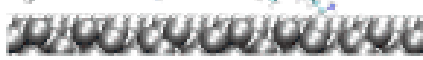

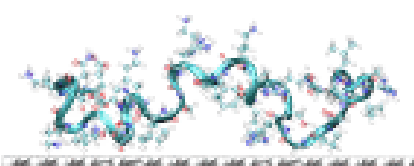

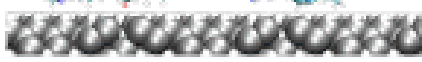

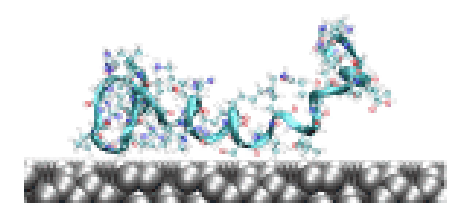

$14 \mathrm{~ns}$

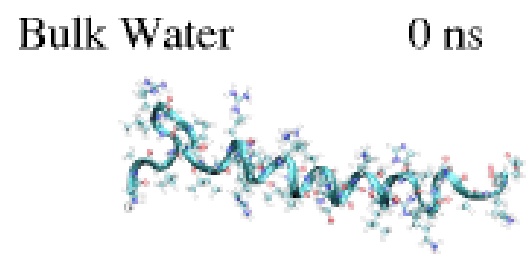

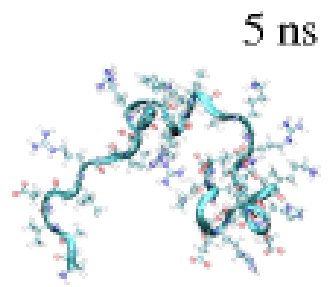

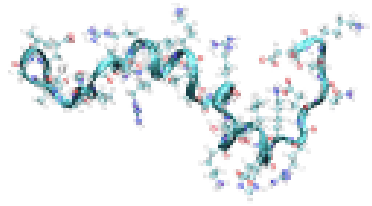

Figure 1, D. J. Cole et al. 


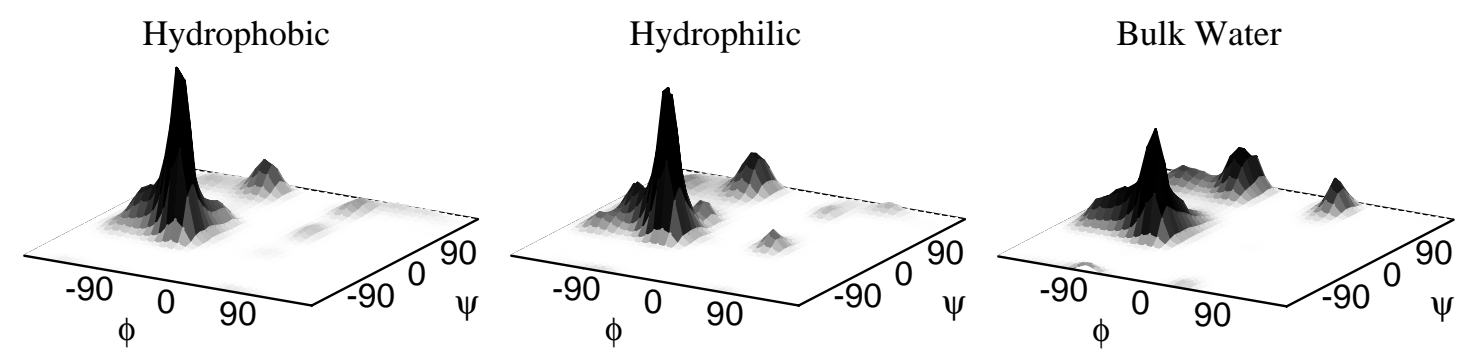

Figure 2, D. J. Cole et al. 

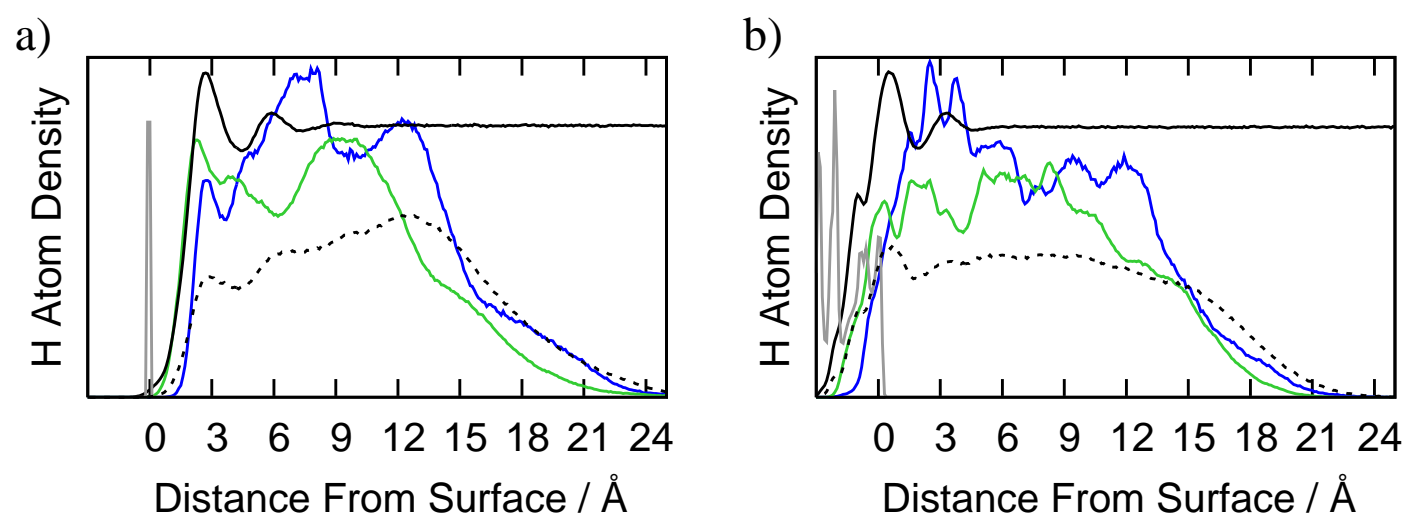

Figure 3, D. J. Cole et al. 
Hydrophobic Hydrophilic Bulk

$\begin{array}{llll}(1) & (2) & (1)\end{array}$

\begin{tabular}{cccccc}
\hline \hline Protein & 98 & 84 & 118 & 126 & 80 \\
Surface & 37 & 36 & $157 / 214$ & $149 / 200$ & - \\
\hline
\end{tabular}

Figure 4, D. J. Cole et al. 